\title{
TERMISUL HOJE: AMPLIANDO HORIZONTES E COMPARTILHANDO CONHECIMENTOS
}

\author{
Cleci Regina Bevilacqua \\ Patrícia Chittoni Ramos Reuillard*
}

\begin{abstract}
Resumo: Este texto presenta los avances llevados a cabo por el Grupo Termisul en lo que se refiere a la investigación teórica y aplicada en Terminología. Después de varios años dedicados sobre todo a la formación de sus investigadores en los estudios terminológicos y de la etapa de elaboración de diccionarios y glosarios en formato tradicional referentes a la temática del medio ambiente, se inició una nueva etapa de actividades e investigaciones en la que el foco principal es compartir los conocimientos adquiridos y el material recogido a lo largo de los años. Con este propósito, se ofrece un ambiente virtual de aprendizaje en el que se encuentran, de forma dinámica, accesible y gratuita, los recursos del Grupo. Se busca, así, complementar y dar continuidad a la formación de los estudiantes de Letras y del futuro profesional interesado en Terminología, redacción y traducción de textos especializados.
\end{abstract}

Palavras-clave: terminología, bases textuales, educação a distancia, combinatórias léxicas especializadas

\section{INTRODUÇÃO}

O Grupo Termisul completa a maioridade em 2011 e se, nos primeiros anos, dedicava toda sua atenção a elaborar glossários e dicionários relativos ao meio ambiente e a aprofundar as bases teóricas que alimentavam o seu fazer, agora seu foco principal é compartilhar os conhecimentos adquiridos e o material coletado. Ao fim da primeira década do novo século, tão marcada pelas tendências de abertura de oferta de formação e inclusão social, o grupo entende como sua essa missão. Isso não significa, no entanto, que, nos primeiros 15 anos, a preocupação com o estudante de Letras e em especial com os interessados na Terminologia tenha sido esquecida. Prova disso são os numerosos cursos de extensão ministrados em nossa Universidade, no Estado do Rio Grande do Sul, no país e no exterior, em que divulgamos os estudos terminológicos e incentivamos muitos a abraçá-los. Ao mesmo tempo, a publicação de obras terminográficas contribuiu para a difusão da terminologia jurídica ambiental que, no Brasil e no mundo, começava a se consolidar. Além disso, mesmo no afã do desenvolvimento da pesquisa aplicada, a formação dos participantes da equipe, professoras e bolsistas, nunca foi descurada. Os primeiros 20 anos se caracterizaram por uma profunda reflexão que resultou em intensa pesquisa e produção teórica (Krieger, neste volume).

Essa reflexão permitiu pensar e tratar temas como o estatuto e as características da terminologia do Direito Ambiental e as propriedades discursivas dos textos legais (KRIEGER et $a l, 2001$ ), as especificidades dos termos jurídicos (MACIEL, 2001), a definição terminológica (FINATTO, 2001), a fraseologia especializada (BEVILACQUA, 1996, 2004), a equivalência na neologia lacaniana (REUILLARD, 2007) e os princípios da Terminologia Textual (KRIEGER, 2008), dentre tantos outros.

\footnotetext{
"Professoras da Universidade Federal do Rio Grande do Sul, Brasil, Departamento de Línguas Modernas e do Programa de Pós-
} Graduação em Letras, membros do Grupo TERMISUL e do Núcleo de Estudos de Tradução Olga Fedossejeva. 
Seguindo o caminho trilhado desde o início, mas buscando ao mesmo tempo inovar, iniciamos uma nova etapa em 2006, que marca uma inflexão considerável nos rumos do Projeto. Deixamos de priorizar a divulgação de materiais de forma mais tradicional, em formato papel, para disponibilizar - de forma dinâmica, acessível e gratuita - todos os recursos construídos no decorrer de tantos anos de pesquisa. Pode-se dizer que passamos a privilegiar a socialização ampla e irrestrita do conhecimento acumulado e a contribuir, através de propostas de ensino a distância, para a formação de estudantes dos Cursos de Letras, profissionais da área e demais interessados não familiarizados com os avanços atuais da pesquisa terminológica teórica e aplicada.

Nesse sentido, é importante destacar dois focos de atenção do grupo. O primeiro é o compromisso acadêmico de oferecer não apenas o material reunido ao longo de tantos anos de pesquisa - sobretudo os textos utilizados para a coleta dos termos que conformam as três obras publicadas pelo grupo - mas também as ferramentas informatizadas, desenvolvidas ao longo das pesquisas, e a produção teórica da equipe. A finalidade é oferecer recursos que possibilitem, por um lado, a busca de dados e informações linguísticas para o desenvolvimento de outras pesquisas e estudos não só no âmbito da Terminologia, mas também da Tradução, do Ensino de Línguas e da Linguística de Corpus e, por outro, a formação e reflexão acadêmicas nessas mesmas áreas.

Essa nova etapa caracteriza-se principalmente pela organização de corpora textuais e pelo desenvolvimento de um sistema de recuperação de dados por meio de ferramentas informatizadas. Assim, a apresentação de diferentes resultados de estudos relacionados entre si, acessíveis on-line e com acesso gratuito e com instruções de uso em língua portuguesa, transformou a página do Termisul na Internet em um verdadeiro ambiente virtual de aprendizagem para o usuário interessado em estudos do texto e do léxico especializados.

Nesse propósito, mantendo o objetivo principal de oferecer tais recursos, nosso segundo foco é o usuário do material disponibilizado. Por essa razão, procuramos construir um uma interface amigável, que facilite a busca rápida e eficaz de informações e que leve em conta as dificuldades do consulente não-informatizado (Maciel, neste volume). Igualmente, elaboramos Objetos de Aprendizagem (OAs), que visam à formação continuada dos interessados em Terminologia e áreas afins.

Outrossim, retomando temas já estudados pelos membros do grupo, mas que demandavam uma análise mais aprofundada, iniciamos, em 2010, um projeto direcionado ao estudo das Combinatórias Léxicas Especializadas (CLEs). Pensamos que esse tema é de grande interesse não apenas porque as CLEs refletem as formas de dizer de determinado âmbito do conhecimento, mas pela necessidade de identificá-las, descrevê-las e registrá-las em bases de dados que estejam ao alcance dos usuários, principalmente os tradutores, redatores e revisores de textos.

Expostos os horizontes de nossa atuação, bem como os focos norteadores das atividades desenvolvidas mais recentemente, apresentamos a seguir os projetos que refletem esse encaminhamento: Acervo Termisul: padrões da linguagem legal, normativa e científica ${ }^{1}$, Combinatórias léxicas especializadas da linguagem legal, normativa e científica e os projetos relacionados à educação a distância ${ }^{2}$, vinculados ao projeto Acervo, a saber, Educação Continuada no Acervo Termisul: Iniciação à Pesquisa Terminológica, Acervo Termisul: um objeto de aprendizagem dos padrões léxico-gramaticais e textuais da linguagem legal, normativa e científica e Acervo Termisul: Pesquisa dos padrões da linguagem especializada com corpus e ferramentas computacionais.

\section{PROJETO ACERVO TERMISUL}

\footnotetext{
${ }^{1}$ Projeto de pesquisa (2006-2010) contemplado no Edital de Ciências Humanas e Sociais do CNPq em 2006. As autoras agradecem ao apoio dos seguintes fomentadores: CNPq, FAPERGS e PROPESQ-UFRGS.

${ }^{2}$ Todos contemplados com bolsas nos editais 09, 11 e 12 da Secretaria de Educação a Distância (SEAD) da UFRGS e podem ser acessados no link Educação Continuada do Acervo Termisul <http://www6.ufrgs.br/termisul/educacao_continuada/index.html\#>.
} 
Este projeto centrou-se na concepção e na publicação de uma nova apresentação para o website do grupo no URL <www6.ufrgs.br/termisul>. A idéia básica era fornecer informações sobre usos, características e práticas textuais, em diferentes idiomas, das linguagens legal, normativa e técnico-científica, de modo a oferecer ao usuário informação qualificada e confiável sobre essas diferentes linguagens, além de instruí-lo sobre passos básicos da pesquisa terminológica. Assim, após a finalização dessa pesquisa em 2010, o site passou a oferecer: a) amostras de textos para uma percepção mais aprofundada do modus dicendi associado a cada um dos domínios contemplados (Legislação Ambiental, Gestão Ambiental e Tecno-Ciência); b) ferramentas on-line para a exploração pontual desses textos; c) bibliografia, tutoriais e módulos instrucionais (Objetos de Aprendizagem - OAs), produzidos para situar o usuário nas questões da pesquisa terminológica teórica e prática.

Ao pensar na criação das bases textuais, abrimos o leque temático com o qual vínhamos trabalhando desde o início do projeto - o meio ambiente e suas interfaces, representadas nas Bases Legis e Gestamb - e passamos a incluir outras áreas de interesse, a saber: Cardiologia, Pediatria, Psiquiatria e Enfermagem, que conformam a Base Tecno-Ciência.

Além dos recursos mencionados, que objetivam promover a formação em terminologia, a Biblioteca Virtual que hoje integra o website do Termisul, abriga um extenso conjunto de trabalhos acadêmicos. Nela, é possível encontrar amostras de glossários desenvolvidos por alunos das disciplinas de Terminologia, mapas conceituais, tutorias referentes aos vários recursos informáticos, artigos e apresentações de trabalhos, dissertações de Mestrado e teses de Doutorado dos membros do grupo e de alunos e ex-alunos do Programa de Pós-Graduação em Letras da UFRGS, vinculados à especialidade Teorias Linguísticos do Léxico e à sua linha de pesquisa Lexicografia e Terminologia: relações textuais ${ }^{3}$.

Cabe destacar que, tendo em vista melhor atender o usuário, foi estruturada uma rede hipertextual que prioriza a busca dos materiais e ferramentas oferecidos e ao mesmo tempo apresenta as explicações necessárias para seu entendimento e manipulação. Com esse propósito, a maneira como é organizada e distribuída a informação na página assegura o interrelacionamento dos recursos disponíveis enquanto favorece a navegação autônoma e a seleção dos conteúdos. (Maciel neste volume). Assim, desenvolvemos e ampliamos o oferecimento de recursos, procurando privilegiar várias frentes: o estudo da terminologia e do modus dicendi das áreas e textos incluídos nas bases, a formação continuada, o aprendizado de ferramentas informatizadas de extração terminológica e a reflexão teórica em Terminologia e em áreas afins.

Paralelamente ao desenvolvimento desse projeto guarda-chuva, foram realizadas outras pesquisas relacionadas: Acervo Termisul: Interface Terminologia/Mapas Conceituais/Legislação Ambiental, coordenado por Anna M. B. Maciel; Identificação e descrição das combinatórias léxicas especializadas da Gestão Ambiental em língua portuguesa $e$ em língua espanhola, coordenado por Cleci R. Bevilacqua; Catálogo de Construções Recorrentes em Cardiologia alemão-português, coordenado por Maria José B. Finatto; Equivalentes tradutórios para os neologismos lacanianos, coordenado por Patrícia C. R. Reuillard.

No que tange aos aspectos teóricos que fundamentaram o projeto Acervo, seguimos os princípios norteadores dos trabalhos anteriores, priorizando o embasamento nas perspectivas comunicativa e textual da Terminologia. De fato, acreditamos que descrever a linguagem especializada implica descrever os seus diferentes usos em distintas situações comunicativas e, por essa razão, adotamos referenciais da Linguística Textual (HOFFMANN, 1998; CIAPUSCIO, 2003); da Terminologia Textual (BOURIGAULT, SLODZIAN, 1999; KRIEGER, FINATTO, 2004; KOCOUREK, 1991a e b), da Terminologia Cognitiva (TEMMERMANN, 2000) e da Terminologia Comunicativa (CABRÉ, 1999).

\footnotetext{
${ }^{3}$ Nessa linha de pesquisa, inserem-se todos os pesquisadores e colaboradores do Projeto Termisul.
} 
Esse referencial teórico justifica-se porque, para tratar o objeto de estudo da Terminologia o termo - e reconhecer seu estatuto de unidade léxica especializada, é imprescindível identificar os cenários comunicativos dos domínios em que são utilizados e as características discursivas dos textos em que ocorrem. Assim, para poder reconhecer os termos, e, para além deles, as fraseologias especializadas ou outras formas de dizer dos textos especializados, é preciso determinar e analisar previamente os elementos próprios desse tipo de comunicação. É essa descrição "macro" tanto do domínio quanto dos textos, em suas tipologias e gêneros, que oferece os elementos para a compreensão do funcionamento da(s) linguagem(ns) especializada(s) e, em consequência, do uso e características específicas das unidades léxicas especializadas.

Tal perspectiva lingüística, conforme afirma Krieger (2008, p.6), torna a identificação terminológica muito mais complexa "exigindo uma série de considerações que não podem desconhecer o funcionamento da linguagem e, conseqüentemente, o comportamento das terminologias, tal como ocorria no âmbito da TGT". Por essa razão, a autora conclui:

Em síntese, se uma teoria da Terminologia procura oferecer princípios para apreender o estatuto terminológico de uma unidade lexical, os componentes cognitivos, lingüísticos e pragmáticos da comunicação especializada ganham relevância. Na mesma linha, o reconhecimento dos mecanismos que engendram a organização narrativa dos textos e dos discursos, conforme a visão semiótica greimasiana, são elementos que auxiliam largamente a identificar o estatuto terminológico de um item lexical. Se uma unidade lexical ganha estatuto de termo especializado no universo de discurso em que se insere, logo, descrever esse universo é etapa metodológica indispensável no quadro dos procedimentos de identificação dessas unidades. (KRIEGER, 2008: 6-7)

Complementando esse quadro, adotamos também os pressupostos teórico- metodológicos da Linguística de Corpus. Ao fazê-lo, buscamos não apenas constituir corpora para a extração de informações e dados lingüísticos, mas também descrevê-los, tal como afirmamos acima, para entender o funcionamento dos universos discursivos e dos textos das áreas com as quais temos trabalhado, não esquecendo também as diferenças entre as diversas línguas.

O pressuposto básico da pesquisa é que a observação extensiva em acervos textuais, nos moldes da Linguística de Corpus (BERBER SARDINHA, 2004) e o respectivo reconhecimento de sua configuração lexical são suscetíveis de fornecer evidências sobre a configuração textual e léxico-gramatical da linguagem científica que estudamos.

A partir das referências anteriores, é possível afirmar que constituir as bases textuais em formato de corpora, criar as ferramentas para extração de informação linguística, reunir os trabalhos produzidos pela equipe, preparar tutoriais sobre os diferentes programas informáticos elaborados e/ou utilizados e ainda pensar numa forma de disponibilizar on-line esse material representou muito mais do que horas incontáveis de dedicação e trabalho dos pesquisadores e dezenas de bolsistas de iniciação científica; representou o início de uma etapa diferenciada, em que se buscou ampliar o impacto social da pesquisa realizada, divulgando de forma mais ampla, mais ágil e gratuita os conhecimentos produzidos pelo grupo, com vistas a oferecer materiais e recursos para o desenvolvimento de outros estudos terminológicos e textuais. Pensamos que, no cenário brasileiro, oferecer esse tipo de informação é de grande valia, se considerarmos o ainda recente desenvolvimento da Terminologia no país e a necessidade de ampliação e aprofundamento dos estudos na área e de sua interface com outras áreas.

Nesse direcionamento, buscando preencher lacunas ainda existentes e aproveitar o material reunido e processado pelo Termisul, focalizamos igualmente a formação continuada em Terminologia e criamos três Objetos de Aprendizagem (OAs). Essa proposta baseia-se nos pressupostos teóricos propostos por Vigostky (1987, 1988), Ausubel (1963) e Moreira (1997).

A partir dos pressupostos sociointeracionistas apresentados por Vygotsky, surge o conceito de aprendizagem significativa (AUSUBEL, 1963), definido como o processo através do qual um novo conhecimento se relaciona de maneira não arbitrária e substantiva (não-literal) à 
estrutura cognitiva do aprendiz (MOREIRA, 1997). Um dos recursos para o desenvolvimento dessa aprendizagem significativa são os OAs, definidos como recursos digitais modulados reutilizáveis, que ajudam o usuário a construir seu conhecimento em determinado campo do saber. Para tanto, colocam à sua disposição os pressupostos básicos da área temática escolhida, orientam a realização de tarefas e oferecem ferramentas que permitem ao aprendiz criar novas atividades, estimulando-o a perseguir novos objetivos.

A partir desses pressupostos, desenvolvemos três projetos relacionados à Educação a Distância, como vem explicado logo a seguir. O primeiro deles - Educação Continuada no Acervo Termisul: Iniciação à Pesquisa Terminológica - teve como objetivo a construção de três OAs a partir de material disponibilizado no Acervo Termisul. Esses OAs refletem os seguintes objetivos: 1) disponibilização dos corpora textuais da Legislação Ambiental Brasileira (Base Legis), da Gestão Ambiental (Base Gestamb) e textos técnico-científicos (Base Tecno-Ciência), a fim de aprimorar os recursos oferecidos nas bases; 2) construção de mapas conceituais da área do Direito Ambiental; e 3) construção de uma base de combinatórias léxicas especializadas da Gestão Ambiental.

Além desses objetos, elaboramos também um mapa conceitual dos conteúdos oferecidos no site do Termisul para facilitar a compreensão por parte do usuário da organização da página e tornar seu acesso ágil e eficaz.

$\mathrm{O}$ segundo projeto - Acervo Termisul: um objeto de aprendizagem dos padrões léxicogramaticais e textuais da linguagem legal, normativa e científica - visou à criação de módulos instrucionais, fundamentados em princípios teóricos da pesquisa empírica da linguagem especializada com corpora digitalizados e ferramentas computacionais, com vistas a operacionalizar o OA elaborado no projeto anterior, oferecer subsídios para a construção do conhecimento teórico da pesquisa linguística com corpus e oportunizar o uso de ferramentas básicas informatizadas e sua aplicação em projetos personalizados. Tais objetivos foram concretizados pela construção de dois módulos instrucionais: 1) de apresentação de pressupostos teórico-metodológicos fundamentais da Terminologia e análise e discussão de sua aplicação contextualizada em relatos de pesquisa documentados on-line, no Acervo Termisul e em outras instituições voltadas para os estudos terminológicos; 2) de aplicação dos pressupostos na construção de produtos terminográficos, tais como glossários e bases de dados.

Finalmente, o terceiro projeto - Acervo Termisul: Pesquisa dos padrões da linguagem especializada com corpus e ferramentas computacionais - teve como meta dar continuidade ao desenvolvimento dos módulos instrucionais, tal como feito no projeto recém mencionado. Construímos outro OA, contendo dois módulos que apresentam novos conteúdos relacionados ao uso de corpora textuais para o estudo da das linguagens especializadas. Os objetivos foram: a) oferecer recursos que permitam a analisar alguns corpora textuais e ferramentas disponíveis on-line e conhecer pesquisas que utilizaram os princípios da Linguística de Corpus; b) utilizar ferramentas de extração de informação linguística e analisar os dados obtidos. Tais objetivos se concretizaram nos módulos seguintes: 1) uso e análise de corpora textuais; 2) prática de ferramentas informatizadas.

Esses projetos de Educação a distância, assim como o Acervo Termisul, dirigem-se aos interessados nos estudos baseados em corpora e léxico especializado, como autores de glossários, tradutores, professores de línguas instrumentais, redatores técnicos e científicos, documentalistas, bibliotecários e gestores de informação, e buscam oferecer subsídios e novas competências para a capacitação profissional desse público. Além disso, também foram e são utilizados nas disciplinas da Graduação do Curso de Bacharelado em Letras/Tradutor e da PósGraduação, naquelas vinculadas à linha de pesquisa Lexicologia e Terminologia: relações textuais.

Pelos objetivos propostos e módulos oferecidos nos OAs, pode-se ver que eles utilizam os recursos e ferramentas oferecidos no Acervo e, ao mesmo tempo, complementam tais recursos, pois são atividades concretas de utilização desses recursos com fins específicos de formação. Com efeito, ao utilizar os recursos e ferramentas oferecidos no Acervo e complementá-los, os 
OAs operacionalizam atividades concretas e cumprem seu objetivo principal, que é a formação do usuário em Terminologia e áreas afins, acompanhada de uma reflexão qualificada.

Cabe ainda dizer que, embora o projeto Acervo Termisul e os OAs a ele relacionados tenham sido concluídos em 2010 e atingidos os objetivos a que se propuseram, as ferramentas são permanentemente revistas e aprimoradas, o que exige de toda a equipe um esforço continuado e enseja novas reflexões.

Mantendo nossa política e o propósito de reutilização do vasto material coletado e de disponibilização de recursos de forma acessível, iniciamos, em agosto de 2010, um novo projeto, que descreveremos a seguir.

\section{COMBINATÓRIAS LÉXICAS ESPECIALIZADAS NA LINGUAGEM LEGAL, NORMATIVA E CIENTÍFICA}

Este projeto tem como objetivo o estudo das expressões multivocabulares recorrentes nas linguagens especializadas, centrando-se em questões referentes à sua caracterização e identificação. Tal estudo permite, por um lado, o oferecimento de produtos terminográficos que contenham tais expressões e, por outro, proporciona significativos avanços no seu reconhecimento automático em aplicações de Processamento da Língua Natural. Os resultados dessa pesquisa subsidiarão principalmente as atividades dos profissionais do texto, como tradutores, redatores de textos técnicos e professores de língua.

Seu objetivo principal é a identificação, descrição e organização em uma base de dados das Combinatórias Léxicas Especializadas (CLEs), a ser disponibilizada na página do Termisul. Tal como os outros projetos, insere-se na perspectiva comunicativa e textual da Terminologia e segue os pressupostos da Linguística de Corpus.

Nesse contexto teórico-metodológico e baseados em Gouadec (1994), L'Homme (2000) e Bevilacqua (1996, 2004), entendemos como CLEs as unidades sintagmáticas recorrentes nas situações de comunicação de áreas temáticas que revelam uma preferência marcante pelas especificidades e pelas convenções próprias do idioma, da área e/ou do gênero textual em que ocorrem. São colocações e fraseologismos que não podem ser explicados unicamente por exigências gramaticais, estilísticas e afinidades semânticas, pois resultam de uma seleção restritiva condicionada ao modo de dizer característico de cada âmbito do conhecimento. Somente a repetição freqüente e o consenso de uma comunidade de falantes que compartilham uma mesma especialidade justificam seu uso. Para o tradutor ou redator, seu conhecimento é indispensável para a produção de textos corretos do ponto de vista lingüístico e adequados do ponto de vista da convencionalidade da área especializada. É fundamental para a produção ou tradução de textos especializados saber, por exemplo, que, para o termo água, há co-ocorrentes como reuso e reaproveitamento, que indicam processos no âmbito de Gestão Ambiental, ou que se diz preservação ou proteção do meio ambiente e não cuidado com o meio ambiente.

Importa observar que, para este projeto, além das línguas já contempladas nos anteriores português, alemão, espanhol, francês e inglês - incluímos a língua italiana, avançando um pouco mais em relação à tradição plurilinguística do grupo.

O projeto prevê a coleta das CLEs nas bases textuais (já mencionadas no projeto anterior) e, por esta razão, divide-se em três grandes etapas, cada uma correspondente a uma base textual do Acervo Termisul. A primeira, de agosto de 2010 a julho de 2012, compreende a coleta, análise e registro das CLEs da Base Legis; a segunda, de agosto de 2012 a janeiro de 2014, tratará das CLEs da Base Gestamb; a terceira e última etapa, de fevereiro de 2014 a julho de 2015, se debruçará sobre as CLEs da Base Tecno-ciência (Cardiologia).

O projeto encontra-se na etapa de coleta das CLEs da Base Legis, que exige previamente a revisão do corpus da Legislação Ambiental Brasileira e nas línguas estrangeiras, a ampliação de alguns corpora, julgados reduzidos para os propósitos atuais, e a constituição do corpus em 
língua italiana. Tal tarefa, já em fase de conclusão, otimizará a coleta das combinatórias, evitando ruídos que possam perturbar sua identificação.

Mesmo tendo estabelecido uma definição para esse tipo de combinatória e critérios para seu reconhecimento (BEVILACQUA et al, 2010), constatamos que, por sua complexidade, tal processo requer não apenas ferramentas mais eficazes, mas também o refinamento dos critérios para sua identificação. Ao iniciar o projeto, começamos a extração de candidatos a CLEs utilizando a ferramenta extrator de $n$-gramas do Acervo, mas observamos que os resultados obtidos geravam muito ruído, isto é, uma quantidade considerável de estruturas que não podiam ser consideradas CLEs no sentido estabelecido pelo grupo, como já referido anteriormente. Fomos levados, então, a testar outros programas, optando pelo uso do AntConc ${ }^{4}$.

Com esse programa e após inúmeras testagens, foi possível observar que a geração de clusters com extensões de 5 a 8 gramas e frequência três oferecia melhores resultados. A partir dos resultados obtidos, percebemos a necessidade de melhorar nossas próprias ferramentas: o extrator de n-gramas foi então aperfeiçoado, oferecendo a possibilidade de extração de clusters com diferentes extensões e freqüências. Da mesma forma, estamos atualizando as ferramentas do corpus paralelo (textos de documentos internacionais relativos ao meio ambiente e da nossa constituição de 1988 traduzida para nossas línguas de trabalho), uma vez recorremos a esse corpus para a busca preliminar dos equivalentes em línguas estrangeiras das CLEs encontradas em português. Este processo está em andamento e sua metodologia e as ferramentas suporte estão em fase de testagem pela equipe.

Apesar dessas dificuldades e dos aperfeiçoamentos iniciais, próprios de um projeto de pesquisa, já temos alguns resultados a apresentar. Assim, a partir da extração de candidatos a CLEs com o uso de nossas ferramentas e da aplicação dos critérios estabelecidos pelo grupo, identificamos um conjunto de CLEs em português, a que chamamos de jurídico-legislativas. Como exemplos, citamos entre outras: no que couber, para os efeitos deste decreto, de acordo com a lei, conforme o disposto no artigo.

A partir da coleta dessas CLEs, iniciamos a busca dos equivalentes nas línguas estrangeiras, processo complexo em si e ampliado quando inclui cinco línguas. Para essa etapa, também nos apoiamos em nossas ferramentas. Partimos da busca de possíveis candidatos a equivalentes no corpus paralelo e validamos os dados obtidos nos corpora da legislação ambiental de cada língua e em outras fontes confiáveis de consulta.

Por entendermos a equivalência (BEVILACQUA, FINATTO, REUILLARD, 2009) como a correspondência conceitual entre um termo ou CLE em língua portuguesa e outro termo ou CLE em língua estrangeira, utilizados em contextos semelhantes, esses equivalentes devem ser oriundos dos mesmos gêneros textuais de partida.

Ao adotar essa definição, é preciso identificar e coletar os contextos de ocorrência das combinatórias para poder realizar uma análise contrastiva ampla do uso das CLEs, que considere os aspectos pragmáticos, semânticos e sintáticos, seguindo os princípios da Terminologia Comunicativa e Textual. É essa etapa que estamos iniciando e que já está proporcionando alguns resultados e vários elementos para discussão. Por exemplo, a perspectiva que cada língua adota ao selecionar determinada CLE em um texto legislativo. Citemos, a título de ilustração, as CLEs se couber/quando couber/no que couber. No texto legislativo em língua portuguesa, o sentido predominante é o de condição, ou seja, se for/caso seja cabível/pertinente $x$, como se vê em "O disposto nesta seção aplica-se, no que couber, às demais cooperativas, inclusive às destinadas a atividades extrativas." Em língua francesa, entretanto, parece vigorar a noção de necessidade, pois a expressão en tant que de besoin remete a dans la mesure où l'on en a besoin [na medida em que se precisa de x], como em "la liste est fixée par arrêtés conjoints du ministre chargé de la protection de la nature et, en tant que de besoin, du ou des ministres compétents, s'ils en font la demande, doivent faire l'objet d'une autorisation délivrée dans les conditions et selon les modalités fixées par un décret en Conseil d'Etat."

\footnotetext{
${ }^{4} \mathrm{O}$ aplicativo AntConc pode ser acessado à página http://www.antlab.sci.waseda.ac.jp/software.html.
} 
Nesse projeto, recém iniciado, temos um longo caminho ainda a percorrer, conforme mostramos ao mencionar todas as etapas previstas. Pensamos que alguns resultados iniciais já foram alcançados, mas sabemos que, na trajetória futura, novos elementos podem surgir e nos surpreender, como sói ocorrer quando se faz pesquisa e, mais ainda, quando essa pesquisa envolve diferentes textos e áreas e diferentes línguas, tudo dirigido a um objeto complexo em sua essência, as CLEs.

\section{CONCLUSÕES}

Ao longo deste artigo, procuramos mostrar a trajetória percorrida pelo Grupo Terminológico Termisul nos últimos vinte anos, salientando o lugar ocupado por esse grupo de pesquisa não somente em sua universidade de origem, mas também no cenário científico nacional e internacional. Evidenciamos que as pesquisas ali desenvolvidas vêm acompanhando par a par o desenvolvimento dos estudos lexicográficos e terminológicos, além de ampliar e incentivar o compartilhamento de sua produção teórica e aplicada.

Tal compartilhamento, que se operacionaliza na oferta on-line dos resultados das pesquisas efetuadas e dos produtos terminográficos para uma ampla gama de usuários, responde à necessidade de um maior impacto social do que é realizado intramuros.

Pode-se igualmente observar que atuamos em várias frentes de trabalho: ampliação da oferta de recursos on-line - buscando apoio tanto em fontes externas quanto criando novos recursos; pesquisa de novos objetos de estudo como as CLEs, consolidando assim nosso entendimento de que as combinatórias léxicas especializadas também são objeto da Terminologia; e, por fim, a formação a distância, dado que buscamos atender usuários aprendizes de tradução e de produção textual do curso de Letras e igualmente profissionais das áreas que têm o texto como matéria de trabalho. Essas novas frentes, por um lado, solidificam os princípios iniciais que nortearam o fazer científico e acadêmico que caracterizaram o grupo desde o seu início e, por outro, abrem novas possibilidades de investigação e oferecem novos temas para discussão e reflexão.

A isso se soma o aproveitamento dos resultados teóricos em novas pesquisas para a formação de futuros pesquisadores, oriundos dos programas de iniciação científica e dos estudos de pós-graduação.

Finalmente, podemos afirmar que a atuação do Termisul contribuiu e continua contribuindo para divulgar e consolidar a Terminologia em nosso país, papel que lhe cabe como um grupo de pesquisa, principalmente quando sediado em uma universidade pública e contemplado com bolsas de pesquisa.

\section{REFERENCIAS}

AUSUBEL, D.P. The psychology of meaningful verbal learning. New York, Gruneand Stratton, 1963.

BERBER SARDINHA, T. Lingüística de Corpus. Barueri-SP: Manole, 2004.

BEVILACQUA, C. R. A fraseologia jurídico-ambiental. Dissertação de Mestrado. Porto Alegre: Universidade Federal do Rio Grande do Sul, 1996.

BEVILACQUA, C. R. Unidades Fraseológicas Especializadas Eventivas: descripción y reglas de formación en el ámbito de la energía solar. Tese de Doutorado Barcelona: Universitat Pompeu Fabra, Institut Universitari de Lingüística Aplicada, 2004.

BEVILACQUA, C. R, et al. (2010). Combinatórias Léxicas Especializadas: etapas prévias para identificação e tratamento. Anais do XII Simpósio Ibero-americano de Terminologia. Buenos Aires: Colegio de Traductores Públicos de la Ciudad de Buenos Aires, Rede Ibero-americana de 
Terminologia. 12 a 16 de setembro de 2010. (no prelo)

BEVILACQUA, C.R; FINATTO, M.F.B.; REUILLARD, P. C. R. (2009). Glossário de Gestão Ambiental: estabelecimento de equivalentes em alemão, espanhol e francês. Tradução \& Comunicação. Revista Brasileira de Tradutores, 19, 2009. p. 61-72.

BOURIGAULT, D.; SLODZIAN, M. Pour une terminologie textuelle. Terminologies Nouvelles, n.19, déc. 1998-juin 1999. p.29-32.

CABRÉ, M. T. La Terminologia. Representación y comunicación. Barcelona: Universitat Pompeu Fabra, Institut Universitari de Lingüística Aplicada, 1999.

CIAPUSCIO, Guiomar. Textos especializados y terminología. Barcelona: Universitat Pompeu Fabra, Institut Universitari de Lingüística Aplicada, 2003.

FINATTO, M. J. Definição terminológica: fundamentos teórico-metodológicos para sua descrição e explicação. Tese, Doutorado. Porto Alegre: Universidade Federal do Rio Grande do Sul, 2001.

GOUADEG, D. Nature et traitement des entités phraséologiques. Terminologie et phraséologie. Acteurs et amenageurs: Actes de la deuxième Université d'Automne en Terminologie. Paris: La Maison du Dictionnaire, 1994. p. 167-193.

HOFFMANN, L. Llenguatges d'especialitat. Selecció de textos. Barcelona: Universitat Pompeu Fabra, Institut Universitari de Lingüística Aplicada, 1998.

KOCOUREK, Rostislav. La langue française de la technique et de la science. Wiesbaden: Oscar Brandstetter Verlag GMBH, 2ème ed, 1991a.

KOCOUREK, Rostislav. Textes et termes. Meta, v.36. n.1, 1991b. p.71-75.

KRIEGER, M. G. Porque Lexicografia e Terminologia: relações textuais. In: Anais do $8^{o}$ Encontro do CELSUL (Círculo de Estudos Linguísticos do Sul). Pelotas: Educat, 2008.

KRIEGER, M. G.; FINATTO, M.J.B. Introdução à Terminologia: Teoria \&Prática. São Paulo, Contexto, 2004.

KRIEGER, M. G. et al. Dicionário jurídico-ambiental: relações de interlocução. In. KRIEGER, M.G.; MACIEL, A.M.B. (Orgs). Temas de Terminologia. Porto Alegre/São Paulo: Ed.UFRGS/Humanitas, 2001. p. 259-267.

KRIEGER, M. G. et al. Environmental law dictionary: from theory to practice. In. KRIEGER, M.G.; MACIEL, A.M.B. (Orgs). Temas de Terminologia. Porto Alegre/São Paulo: Ed.UFRGS/Humanitas, 2001. p. 268-274.

L'HOMME, M. C. Understanding Specialized Lexical Combinations. Terminology, vol. 6, n. 1, 2000. p. 89-110.

MACIEL, A.M.B. Para o reconhecimento da especificidade do termo jurídico. Tese de Doutorado. Porto Alegre: Universidade Federal do Rio Grande do Sul, 2001.

MOREIRA, M.A. Aprendizagem significativa: um conceito subjacente. In: MOREIRA, M.A., CABALLERO, M.C. e RODRÍGUEZ, M.L. (orgs.). Actas del Encuentro Internacional sobre el Aprendizaje Significativo. España, 1997. p. 19-44.

REUILLARD, P.C.R. Neologismos lacanianos e equivalências tradutórias. Tese de Doutorado. Porto Alegre: Universidade Federal do Rio Grande do Sul, 2007.

TEMMERMANN, Rita. Towards new ways of terminology description. The sociocognitive approach. Amsterdam/New York: John Benjamins, 2000.

VYGOTSKY, L.S. Pensamento e linguagem. São Paulo, Martins Fontes, 1987.

VYGOTSKY, L.S. A formação social da mente. São Paulo, Martins Fontes, 1988. 